Check for updates

Cite this: RSC Adv., 2019, 9, 19699

Received 15th March 2019

Accepted 7th June 2019

DOI: 10.1039/c9ra01987a

rsc.li/rsc-advances

\section{Batch microfabrication and testing of a novel silicon-base miniaturized reference electrode with an ion-exchanging nanochannel array for nitrite determination}

\author{
Jiawen Yin, (D) a Wei Zhang, ${ }^{\text {tb }}$ Zan Zhang, ${ }^{a}$ Han Jin, ${ }^{a}$ Wanlei Gao, ${ }^{a}$ Jiawen Jian $^{\text {a }}$ \\ and Qinghui Jin*ab
}

The reference electrode (RE) provides a stable potential for electrochemical detection; therefore, the RE plays an important role in environmental monitoring. In this paper, a novel batch of microfabricated silicon-base miniaturized $\mathrm{Ag} / \mathrm{AgCl} \mathrm{RE}$ was reported. A specially designed mini-tank for saturated $\mathrm{KCl}$ solution storage and a nanochannel array for ion-exchange were fabricated on a 4 inch (100) silicon wafer using a two-step $\mathrm{KOH}$ anisotropic etching process. $\mathrm{An} \mathrm{Ag} / \mathrm{AgCl}$ electrode was fabricated on a 4 inch Pyrex 7740 glass substrate. Finally, the finished silicon and glass substrates were anode bonded to form the entire system. By comparing with a conventional solid-state $\mathrm{Ag} / \mathrm{AgCl} \mathrm{RE}$ in electrochemical microsensors, a pre-packaged saturated $\mathrm{KCl}$ solution in the mini-tank provided a stable working environment for the $\mathrm{Ag} / \mathrm{AgCl}$ electrode to ensure a constant reference potential. Compared with a routine glass-structured RE and by replacing the ion-exchange membrane with a nanochannel array, the miniaturized RE achieved a longer lifetime. The size of the finished miniaturized RE electrode was $11 \mathrm{~mm} \times 14 \mathrm{~mm}$. The reference potential variation was only $0.1 \mathrm{mV}$ under continuous testing for $3000 \mathrm{~s}$. The standard deviation in the reference potential was only $1.314 \mathrm{mV}$ in different $\mathrm{Na}_{2} \mathrm{SO}_{4}$ buffer concentrations ranging from $3 \mathrm{mM}$ to $30 \mathrm{mM}$. To verify the practicality of the novel silicon-base miniaturized $R E$, the fabricated RE was applied to measure the amount of nitrite in a water sample and achieved a better linearity of $R^{2}=0.998$. This miniaturized RE showed better reference potential stability and consistency because of the batch fabrication technique. This novel strategy for the design and manufacture of the miniaturized RE shows a bright future in the wide use of electrochemical sensors in online monitoring of water pollutants.

\section{Introduction}

Waste production from agriculture, industrial sewage, and animal and human activities is affecting the boundaries between clean water and wastewater, causing a reduction in the fresh water supply for humans. Biological and chemical contaminants in tap and drinking water initiate the spread of contagious diseases. Therefore, fast and sensitive detection techniques are crucial to ensure a safe and clean water supply. Several water monitoring techniques are available, including conventional instrumental analysis (laboratory-based analysis), ${ }^{\mathbf{1 , 2}}$ sensor placement approach, ${ }^{3}$ microfluidic devices, ${ }^{\mathbf{4}, 5}$ spectroscopic approaches, ${ }^{6,7}$ and biosensors. ${ }^{8,9}$ For the online

${ }^{a}$ Faculty of Electrical Engineering and Computer Science, Ningbo University, Ningbo, 315211, P. R. China. E-mail: jinqinghui@nbu.edu.cn

${ }^{b}$ State Key Laboratory of Transducer Technology, Center for Excellence in Superconducting Electronics, Shanghai Institute of Microsystem and Information Technology, Chinese Academy of Sciences, Shanghai 200050, P. R. China. E-mail: wei.zhang@mail.sim.ac.cn; jinqh@mail.sim.ac.cn monitoring demand, electrochemical detection technology is widely used because of its high sensitivity, low cost, high-speed detection, miniaturization, easy integration, and convenient application. ${ }^{\mathbf{1 0 - 1 2}}$ An electrochemical detection system consists of a working electrode (WE), reference electrode (RE), and counter electrode (CE). The RE provides a stable reference potential for the electrochemical detection system to ensure that the potentials of the WE are accurate. Therefore, a RE with excellent performance is the key for the accurate detection of all analytes.

Many types of REs are available, such as a calomel electrode, hydrogen electrode, and silver chloride electrode. ${ }^{13-16}$ In the field of water ion detection, the $\mathrm{Ag} / \mathrm{AgCl}$ electrode is the most common RE. The glass cavity of the electrode has a certain concentration of $\mathrm{KCl}$ solution, and the internal solution is separated from the external water environment using a porous glass. During detection, chloride ions diffuse through the porous film, decreasing the concentration of chloride ions. Because the potential of the electrode satisfies the Nernst equation, a decrease in the concentration of chloride ions 
corresponds to a change in the reference potential. Recently, to develop a more stable, more convenient, and low cost RE, studies on $\mathrm{Ag} / \mathrm{AgCl} \mathrm{RE}$ have mainly focused on the variation in the electrolyte of the electrode, variation in the structure of the $\mathrm{RE}$, and miniaturization and integration of the electrode. Suzukia et al. designed a liquid-junction $\mathrm{Ag} / \mathrm{AgCl} \mathrm{RE}$ using microfabrication techniques. They used a novel thin-film Ag/ $\mathrm{AgCl}$ structure. The container for the internal electrolyte solution was developed by the anisotropic etching of a silicon substrate. A cellulose-acetate-plug junction instead of a salt bridge was used to ensure that the electrode could maintain a potential of $\pm 1 \mathrm{mV}$ over a few hours. ${ }^{17}$ The miniaturization of a $\mathrm{RE}$ is mostly achieved by changing the internal reference liquid, that is, by replacing the liquid electrolyte with a gel electrolyte or an all-solid electrolyte. ${ }^{\mathbf{8 - 2 1}}$ Ciobanu et al. reported the manufacturing and performance of miniaturized REs with wide applicability and highly stable potential in both aqueous and organic media. The REs showed a potential stability of $\pm 0.5 \mathrm{mV}$ over $30 \mathrm{~h}$ and a low-temperature coefficient of $0.15 \mathrm{mV}$ $\mathrm{K}^{-1}{ }^{18}$ The miniaturized RE could be produced with a small size and low cost, but this does not mean that the ions present inside the gel do not flow out. The lifetime of an electrode is still a severe problem; therefore, studies on the salt bridge of REs have continued. ${ }^{22,23}$ Pedrotti et al. designed a high-density microporous polyethylene for a restricted flow junction between the internal and external electrolytes. The more favorable characteristics of a microporous membrane for the fabrication of miniaturized junctions are evident in a commercial Vycor membrane. ${ }^{22}$ Zevenbergen et al. conducted a quantitative study on the relationship between the micropore size and chloride drift. They found that the diameter of the micropores and the loss of chloride ions are linearly related. When the hole diameter was $20 \mu \mathrm{m}$, the potential change was $0.01 \mathrm{mV}$ per day during the testing for two months. ${ }^{23}$ In addition, some special applications and integration studies on $\mathrm{Ag} / \mathrm{AgCl}$ REs have been reported. ${ }^{24-26}$ Hayashi et al. invented a $\mathrm{RE}$ to use in a flow-type measuring apparatus by incorporating an immobilized enzyme WE. ${ }^{24}$ To eliminate the need for a separate RE and facilitate the use of an ion-sensitive field-effect transistor, an allsolid-state RE integrated with ISFET in one chip has been developed. ${ }^{26}$

The role of a RE in an electrochemical system is to provide a stable reference potential under different buffer concentrations during the detection over a long period of time. The reaction on the surface of a $\mathrm{Ag} / \mathrm{AgCl}$ electrode can be expressed as the following two processes in a certain concentration of $\mathrm{KCl}$ solution ${ }^{27}$ :

$$
\begin{gathered}
\mathrm{Ag}^{+}+\mathrm{e}^{-} \leftrightarrows \mathrm{Ag} \\
\mathrm{Ag}^{+}+\mathrm{Cl}^{-} \leftrightarrows \mathrm{AgCl}
\end{gathered}
$$

When the reaction reaches dynamic equilibrium, the potential of the electrode stabilizes to a fixed value. Fig. 2 shows the electrode operation. The potential satisfies the following Nernst equation: ${ }^{28}$

$$
E=E_{0}-\frac{R T}{n F} \ln a_{\mathrm{Cl}^{-}}
$$

$E_{0}$ is the RE standard potential at $25{ }^{\circ} \mathrm{C}, R\left(\mathrm{~J} \mathrm{~K}^{-1} \mathrm{~mol}^{-1}\right)$ is the gas constant, $T\left({ }^{\circ} \mathrm{C}\right)$ is the temperature, $F\left(\mathrm{C} \mathrm{mol}^{-1}\right)$ is the Faraday constant, and $a_{\mathrm{Cl}^{-}}(\mathrm{M})$ is the chloride ion concentration in the internal reference liquid $(\mathrm{KCl})$. The equation shows that the temperature $T$ and chloride ion concentration $a_{\mathrm{Cl}^{-}}$affect the $\mathrm{RE}$ potential. The RE potential is inversely proportional to $T$ and $\ln a_{\mathrm{Cl}^{-}}$:

$$
E=0.2224-0.05916 \lg a\left(\mathrm{Cl}^{-}\right)
$$

Therefore, it is important to maintain the stability of the chloride ion concentration in the internal reference liquid at a constant temperature.

Nutrient salts refer to the inorganic salts of nitrogen, phosphorus, and silicon in seawater. They are essential elements for the growth of marine algae, the primary produce of ocean and the basis of the marine food chain. Excess nutrients can lead to the eutrophication of seawater. This can cause red tides, green tides, and marine pollution, leading to the death of marine life and even leading to air pollution. ${ }^{29}$ Therefore, it is important and necessary to establish a large-scale, real-time, and highprecision nutrient salt detection network to prevent the occurrence of natural disasters.

To achieve the large-scale and real-time detection of the water environment, in this study, a novel silicon-based miniaturized $\mathrm{Ag} / \mathrm{AgCl} \mathrm{RE}$ was designed and batch-fabricated based on the micro and nano-fabrication technology. Compared with the conventional solid-state $\mathrm{Ag} / \mathrm{AgCl} \mathrm{RE}$ used in some electrochemical microsensors, the prepackaged saturated $\mathrm{KCl}$ solution in the mini-tank provides a stable working environment for the $\mathrm{Ag} / \mathrm{AgCl}$ electrode to ensure a constant reference potential. The batch-fabricated miniaturized $\mathrm{Ag} / \mathrm{AgCl} \mathrm{RE}$ has good accuracy, stability, and consistency. It provides a feasible technical solution for the design of detection sensors for water pollutants, making it possible to construct a large-area sensor network for water quality monitoring.

\section{Experimental}

\subsection{Miniaturized Ag/AgCl RE design}

The schematics of the miniaturized $\mathrm{Ag} / \mathrm{AgCl} \mathrm{RE}$ are shown in Fig. 1. Fig. 1(A and D) show the mass production of a novel RE on a 4 inch (100) silicon wafer. A specially designed mini-tank for saturated $\mathrm{KCl}$ solution storage and a nanochannel array for ion-exchange were fabricated on a 4 inch (100) silicon wafer using a two-step $\mathrm{KOH}$ anisotropic etching process (Fig. 1D). ${ }^{30}$ The $\mathrm{Ag} / \mathrm{AgCl}$ electrode was fabricated on a 4 inch Pyrex 7740 glass substrate (Fig. 1B and E). Then, the finished silicon and glass substrates were anode-bonded to form the entire system (Fig. 1C) ${ }^{31}$ The size of a separated single sensor is $14 \mathrm{~mm} \times$ $11 \mathrm{~mm}$ (Fig. 1F). The schematic shows the details of the nanochannel array in Fig. 1G. Fig. 2 shows the working principle of the Ag/AgCl RE. The mini-tank is separated with the outer water sample using a beam with a width of $20 \mu \mathrm{m}, 30 \mu \mathrm{m}$, and $40 \mu \mathrm{m}$. A series of channel arrays with a depth of nanometers for the chloride ion exchange were prepared on the surface of the beam by controlling the duration of $\mathrm{KOH}$ etching. The dimensions of 
the finished nanochannel array are $20-40 \mu \mathrm{m}$ long, $15 \mu \mathrm{m}$ wide, and less than $1 \mu \mathrm{m}$ deep. Compared with a traditional glass tube $\mathrm{RE}$, the nanochannel array of the novel RE can significantly decrease the loss of chloride ions in the $\mathrm{KCl}$ solution and effectively increase the service life of the RE. This shows that the drift rate of a $\mathrm{RE}$ reference potential is proportional to the diameter of the ion exchange channel, as obtained by Zevenbergen et al. ${ }^{23}$ The $\mathrm{Ag} / \mathrm{AgCl}$ electrode with a thickness of about $0.5 \mu \mathrm{m}$ was fabricated on a Pyrex 7740 glass using a silver sputtering lift-off process and electrochemical treatment using a $\mathrm{HCl}$ solution. A hole with a $1.4 \mathrm{~mm}$ diameter was prepared using a laser for injecting a saturated $\mathrm{KCl}$ solution. After dicing, the saturated $\mathrm{KCl}$ reference solution was filled into the minitank to maintain the stability of the RE reference potential. The electrode lead was welded using a conductive silver paste for electric signal collection and sealed with epoxy glue, as shown in Fig. 2. The number of nanochannels were 2, 4, 8, and 12 , and their depths were $380 \mathrm{~nm}( \pm 50 \mathrm{~nm}), 600 \mathrm{~nm}( \pm 50 \mathrm{~nm})$, $840 \mathrm{~nm}( \pm 50 \mathrm{~nm})$, and $1.8 \mu \mathrm{m}( \pm 50 \mathrm{~nm})$, respectively.

\subsection{Batch fabrication process flow of miniaturized RE}

The novel RE was batch-fabricated on a 4 inch (100) silicon wafer and 4 inch Pyrex 7740 glass substrate. The process flow can be divided into two parts as shown in Fig. 3: (i) processing
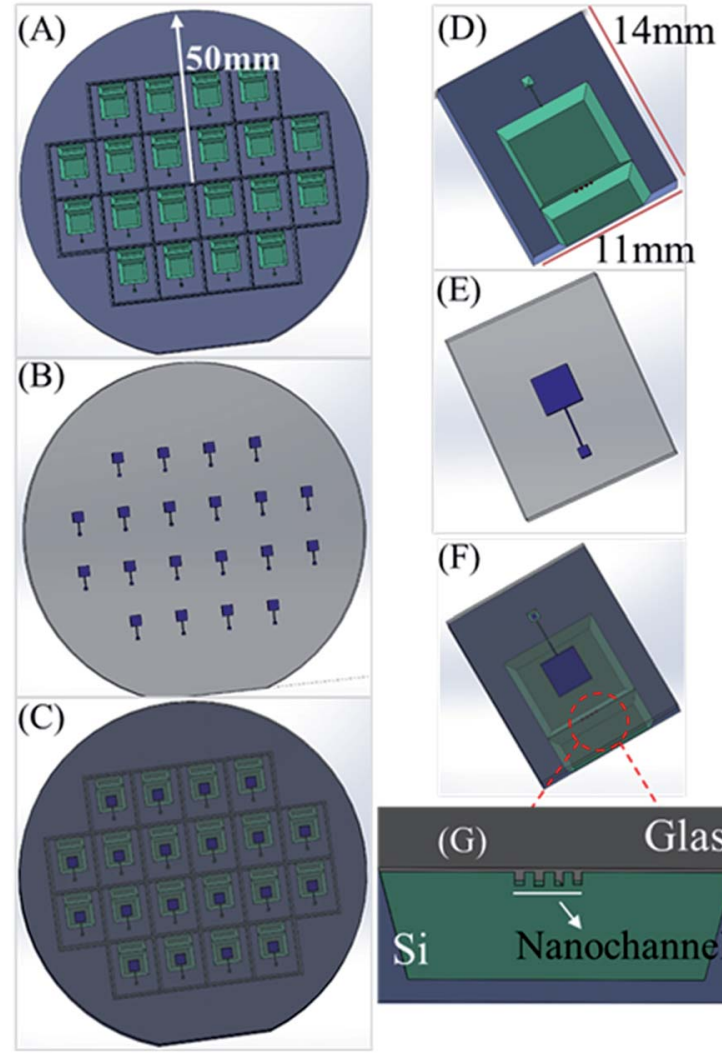

(E)

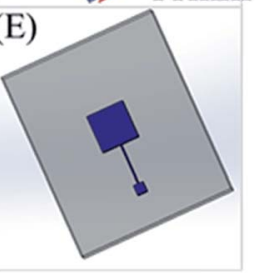

(F)
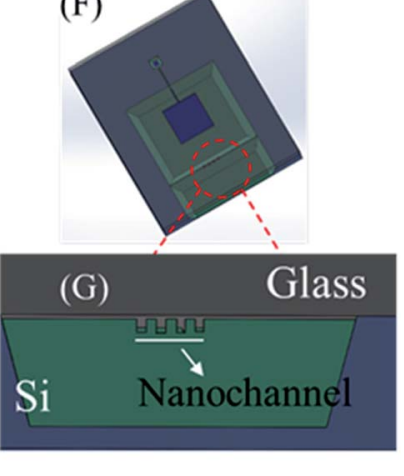

Fig. 1 (A) Batch processing of 4 inch silicon wafer. (B) Batch processing of 4 inch glass. (C) Anodic bonding of 4 inch silicon wafer and 4 inch glass wafer. (D) Anisotropic wet etching specific structure of silicon wafer. (E) Preparation of thin-film $\mathrm{Ag} / \mathrm{AgCl}$ electrodes on glass sheets. (F) Silicon-glass bonding to form a nanochannel array. (G) The details of the nanochannel array in beam.

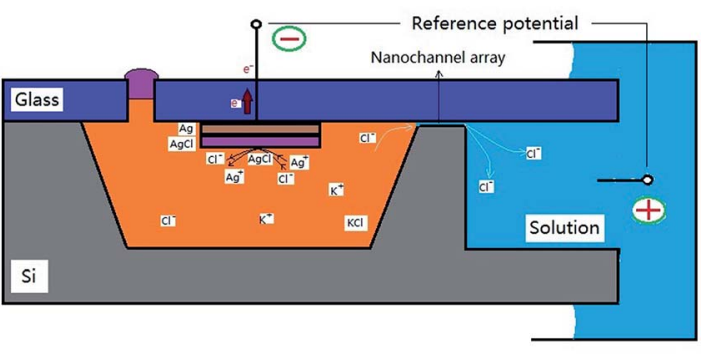

Fig. 2 Working principle diagram of $\mathrm{Ag} / \mathrm{AgCl} \mathrm{RE}$ and a sectional view of the completed electrode chip (Fig. 1F).

the silicon substrate mini-tank and nanochannel array and (ii) preparation of the $\mathrm{Ag} / \mathrm{AgCl}$ electrode on the glass substrate.

2.2.1. Preparation of silicon-based nano channel array and solution storage tank. A double-side-polished 4 inch (100) crystal orientation silicon wafer with about $2 \mu \mathrm{m}$ thickness was selected as the $\mathrm{SiO}_{2}$ layer, as shown in Fig. 3B. The silicon wafer was thoroughly cleaned and dried and then coated with a thickness of $2.4 \mu \mathrm{m}$ LC100A photoresist (Fig. 3C). The wafer was exposed to a lithography machine for $15 \mathrm{~s}$ and then immersed in an FHD-320 solution for $40 \mathrm{~s}$ for patterning

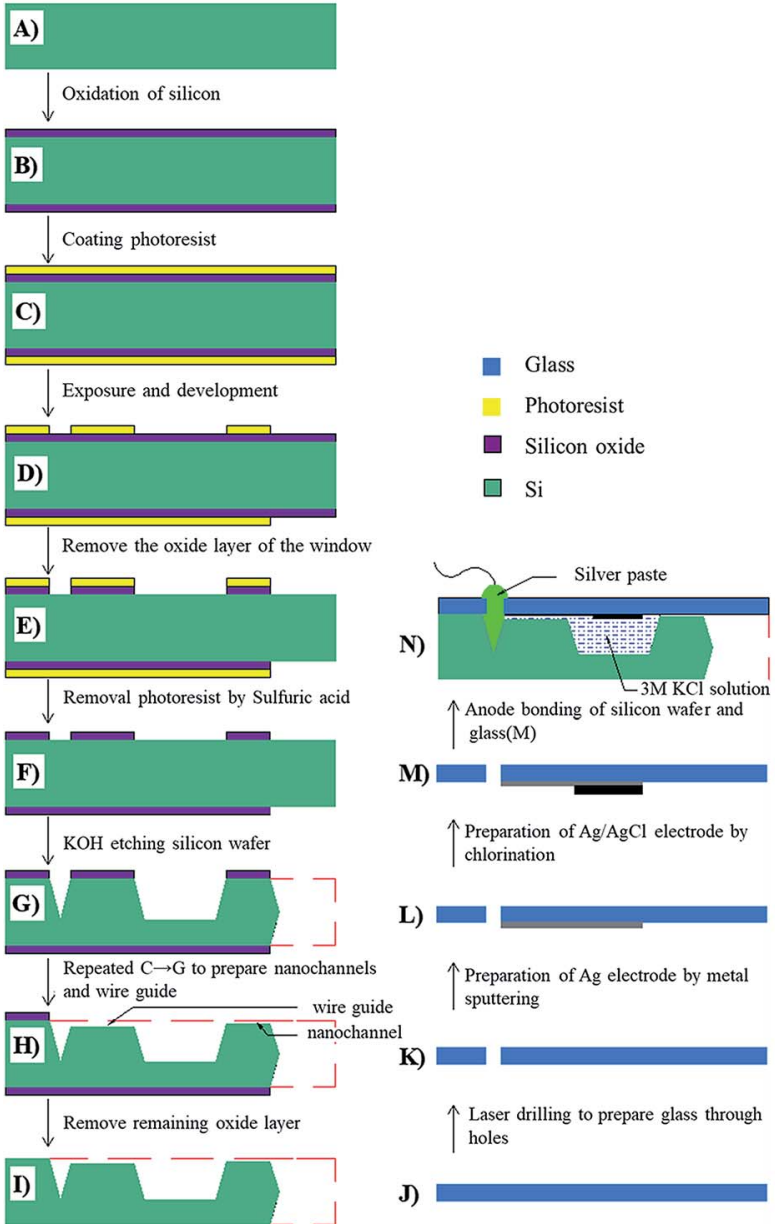

Fig. 3 Batch manufacturing of micro-nano $\mathrm{Ag} / \mathrm{AgCl} \mathrm{RE}$. 


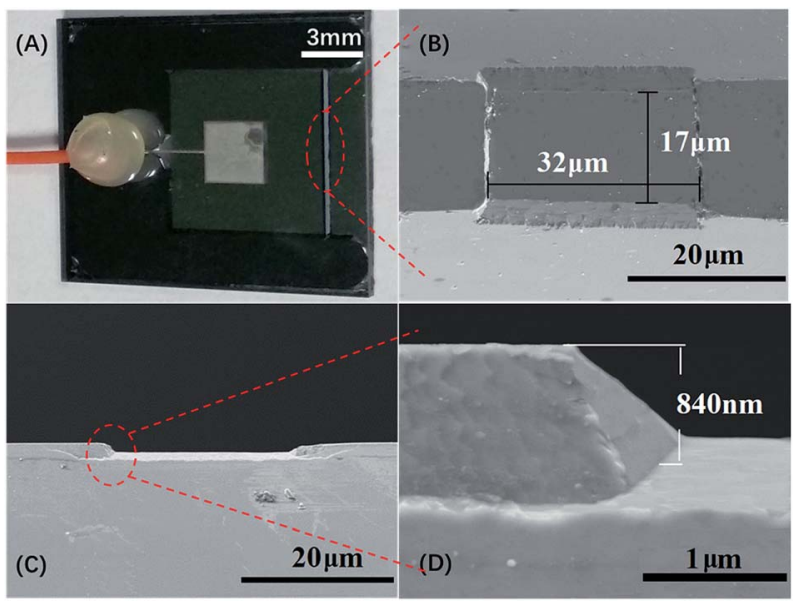

Fig. 4 Photographs and SEM images of micro-nano device: (A) photograph of $\mathrm{Ag} / \mathrm{AgCl}$ reference electrode, (B) top view, (C) crosssection view, and (D) image at zoom in mode of channel.

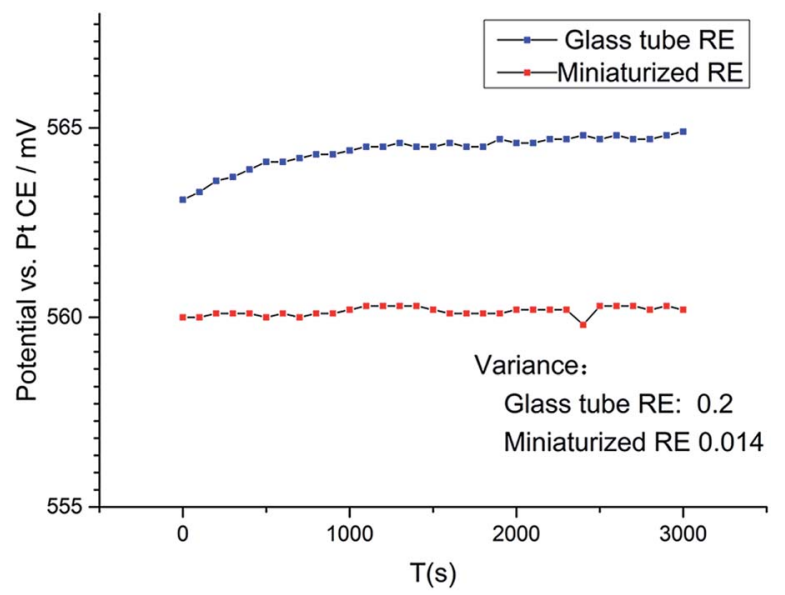

Fig. $525^{\circ} \mathrm{C}$, long-term (3000 s) potential measurement of miniaturized $\mathrm{RE}$ in $3 \mathrm{mM} \mathrm{Na}_{2} \mathrm{SO}_{4}$ buffer solution and comparison with glass tube electrode under the same external conditions.

(Fig. 3D). A buffer oxide etching (BOE) solution was used to completely etch the bare oxide layer for $720 \mathrm{~s}$ for the next $\mathrm{KOH}$ wet etching (Fig. 3F). However, all photoresists should be removed before this step (Fig. 3E). Next, the anisotropic wet etching of the bare drained silicon was carried out with a $\mathrm{KOH}$ solution. ${ }^{30}$ The concentration of the $\mathrm{KOH}$ wet etching solution was $30 \%$. At $50{ }^{\circ} \mathrm{C}$, the $\mathrm{KOH}$ anisotropic etching had an etching rate of $10.3 \mu \mathrm{m} \mathrm{h}^{-1}$ for the (100) silicon layer as compared to that of $0.05-0.06 \mu \mathrm{m} \mathrm{h}^{-1}$ for the $\mathrm{SiO}_{2}$ layer. According to the difference in the etching rate of $\mathrm{Si}$ and $\mathrm{SiO}_{2}$, the mini-tank structure with a depth of $250 \mu \mathrm{m}$ was prepared after about $20 \mathrm{~h}$ of etching (Fig. 3G). Spin coating, aligning, and patterning of the $\mathrm{SiO}_{2}$ layer $\mathrm{BOE}$ etching were repeated for the nanochannel array fabrication and wire guide (Fig. 3H). The time (less than $5 \mathrm{~min}$ ) of $\mathrm{KOH}$ etching was controlled to fabricate the nanochannel array with a depth of less than $1 \mu \mathrm{m}$ on the microbeam, which emerged by the wet etching of silicon on both sides of the beam. The $\mathrm{SiO}_{2}$ layer was removed by $\mathrm{BOE}$

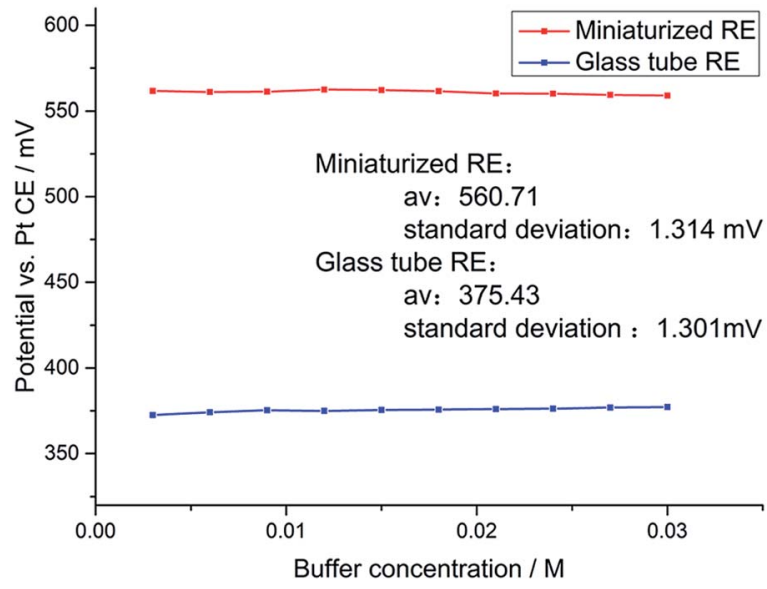

Fig. $625^{\circ} \mathrm{C}$, the potential of the miniaturized reference electrode with sodium sulphate buffer concentration varies from $3 \mathrm{mM}$ to $30 \mathrm{mM}$ compared with a glass tube electrode in the same external conditions.

Table 1 Miniaturized RE stable potential and impedance of samples

\begin{tabular}{llllll}
\hline Numbering & $1 \#$ & $2 \#$ & $3 \#$ & $4 \#$ & $5 \#$ \\
\hline Potential $(\mathrm{V})$ & 0.534 & 0.574 & 0.559 & 0.564 & 0.586 \\
Number of nanochannels & 2 & 4 & 8 & 12 & 12 \\
Impedance $(\Omega)$ & $17.4 \mathrm{k}$ & $15 \mathrm{k}$ & $9.1 \mathrm{k}$ & $3.6 \mathrm{k}$ & $3.4 \mathrm{k}$ \\
\hline
\end{tabular}

etching to prepare the anode bonding with glass substrate. The finished silicon substrate with a mini-tank and nanochannel array is shown in Fig. 3I.

2.2.2. Preparation of $\mathrm{Ag} / \mathrm{AgCl}$ electrode on a glass substrate. The 4 inch Pyrex 7740 glass substrate was cleaned using a standard washing process and $\mathrm{O}_{2}$ plasma treatment. A silver layer with the thickness of about $0.4 \mu \mathrm{m}$ was obtained by sputtering, forming the silver electrode by a lift-off process (Fig. 3L). A circular hole with a diameter of $1.4 \mathrm{~mm}$ was prepared on the glass surface using laser drilling technology to fill the $\mathrm{KCl}$ solution (Fig. 3K). A certain thickness of $\mathrm{AgCl}$ was deposited on the surface of the Ag layer by a $0.25 \mathrm{M} \mathrm{HCl}$ solution electrochemical treatment using a constant voltage of $4 \mathrm{~V}$ (Fig. 3M).

Finally, the silicon wafer and glass substrate were cleaned using a mega-sonic instrument for $30 \mathrm{~min}$, and the entire wafer was anode-bonded together. ${ }^{31}$ After dicing, a saturated $\mathrm{KCl}$ solution was filled into the mini-tank. The lead electrode was welded using a conductive silver paste and sealed with epoxy glue to form the miniaturized Ag/AgCl RE (Fig. 3N).

\subsection{Miniaturized RE testing and nitrite determination}

The microstructure was observed by scanning electron microscopy (SEM). The stability and consistency of the miniaturized RE were evaluated and tested using an electrochemical working station (Gamry Reference 600+). Comparative testing was carried out with the miniaturized $\mathrm{RE}$ and a conventional glass tube Ag/AgCl RE. The open-circuit voltage method was used to test the electrode potential of the RE. A Au disk electrode was 

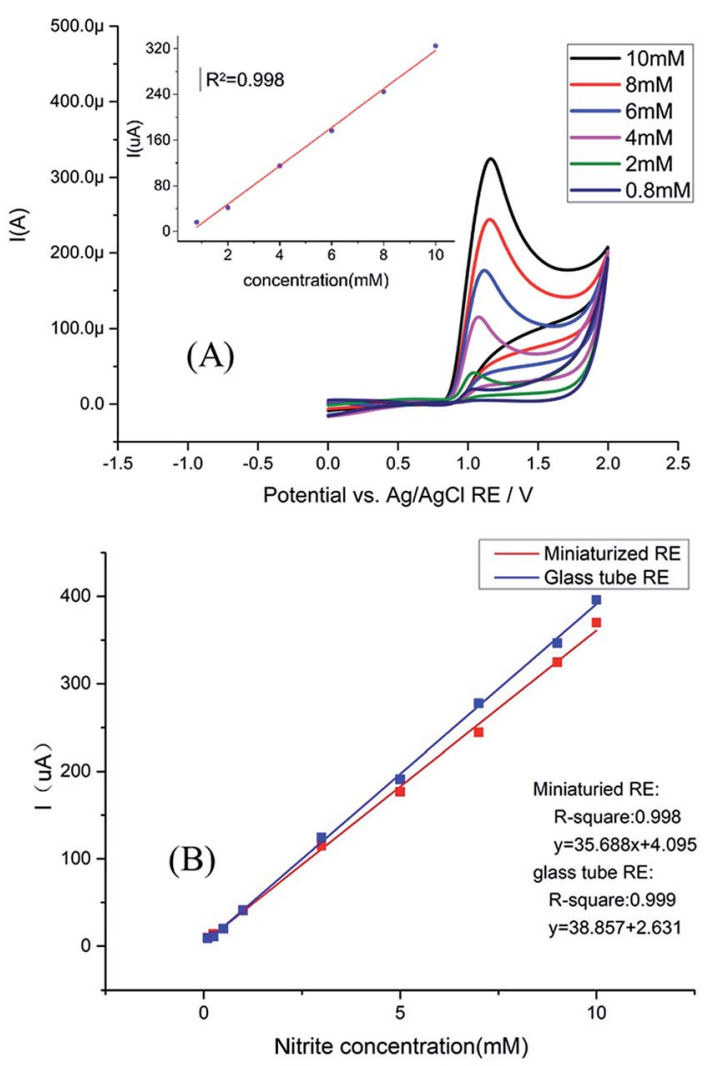

Fig. 7 (A) Gold disk electrode as the WE, Pt electrode as the CE, and miniaturized $\mathrm{Ag} / \mathrm{AgCl} \mathrm{RE}$ as the RE. Determination of nitrite content in water in $0.1 \mathrm{M}$ PBS buffer, $100 \mathrm{mV} \mathrm{s}^{-1}, 25^{\circ} \mathrm{C}, \mathrm{CV}$. (B) Using a miniaturized RE and a glass tube RE to form a three-electrode system: comparison of the linear relationship between concentration and current peaks.

used as the WE, and a Pt electrode was used as the CE. This RE was used to construct a three-electrode system for the detection of nitrite and to verify its feasibility in comparison with a glass tube RE. The amount of nitrite was determined in the concentration range from $800 \mu \mathrm{M}$ to $10 \mathrm{mM}$ using cyclic voltammetry (CV).

\section{Results and discussion}

\subsection{SEM view of nanochannel array}

A typical glass tube RE uses a semipermeable membrane to exchange the internal solution with the external water environment. Previous studies have used high-density microporous polyethylene $e^{22}$ with a single micron hole ${ }^{23}$ instead of the semipermeable membrane of the traditional glass tube $\mathrm{Ag} / \mathrm{AgCl}$ electrode. This study gradually improved the performance of the electrode, especially the life of the electrode. In this study, the specially designed and fabricated nanochannel array in the micro RE replaced the traditional semipermeable membrane as a chloride ion exchange channel for the electrode to achieve good stability and durability. Fig. 4 shows the SEM image of the beam surface channel after $\mathrm{KOH}$ wet etching for $9 \mathrm{~min}$. The height of the channel is $840 \mathrm{~nm}$ and the width is $32 \mu \mathrm{m}$. The length is $17 \mu \mathrm{m}$, and the channel surface is smooth enough for the solution to flow through. The depth of the channel array can be controlled by varying the etch time (about $190 \mathrm{~nm} \mathrm{~min}{ }^{-1}$ ). The surface flatness of the 4 inch silicon wafer and glass sheet after polishing is less than $60 \mathrm{~nm}$, and the flatness of a single electrode chip (the length of the beam is $7 \mathrm{~mm}$ ) is less than $5 \mathrm{~nm}$. After the silicon-glass anodic bonding, the area above the beam is completely sealed except for the nanochannel array.

\subsection{Stability of miniaturized RE under continuous testing for a long time}

Normally, the concentration of chloride ions in a $\mathrm{KCl}$ solution decreases during electrochemical detection due to diffusion motion. According to the Nernst equation, the reference potential will increase over time. The accuracy of detection is affected when the reference potential changes significantly. The drift rate of the RE reference potential is proportional to the diameter of the ion exchange channel, as obtained by

Table 2 Comparison of the miniaturized $\mathrm{Ag} / \mathrm{AgCl} \mathrm{RE}$ with previously reported $\mathrm{Ag} / \mathrm{AgCl} \mathrm{REs}$

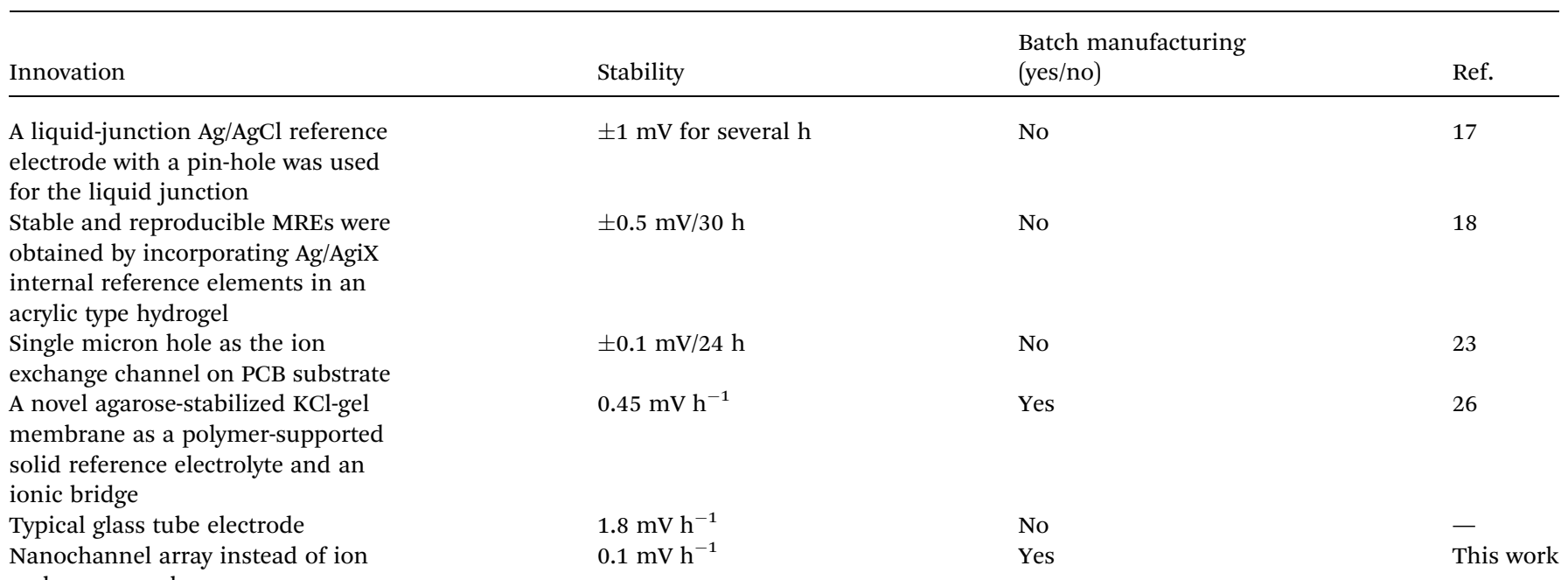


Zevenbergen et al. $^{23}$ Fig. 5 shows the stability of the electrode reference potential under the condition of continuous testing for $3000 \mathrm{~s}$ measured using the open-circuit voltage method in $3 \mathrm{mM} \mathrm{Na}_{2} \mathrm{SO}_{4}$ buffer. The method for measuring the reference potential of the open-circuit voltage is to test the potential between $\mathrm{Ag} / \mathrm{AgCl} \mathrm{RE}$ and an inert electrode $(\mathrm{Pt})$ in the buffer. According to the Nernst equation, a decrease in the concentration of chloride ions in the reference solution increases the reference potential. Fig. 5 shows that the RE has a stable reference potential of $559.8 \mathrm{mV}$. The increment in potential within $3000 \mathrm{~s}$ is only $0.1 \mathrm{mV}$, which is much smaller than $1.8 \mathrm{mV}$ from the glass tube electrode. We can conclude that the chloride ion loss of the miniaturized $\mathrm{RE}$ is slower than the glass $\mathrm{RE}$ from Fig. 5. A less significant drift in the potential means that the miniaturized RE has a longer life. However, the miniaturized RE showed an increment in the potential of about $185 \mathrm{mV}$ compared to the glass RE. The major reason is related to the potential generated by the semiconductor and metal contact. During the RE soldering, the direct contact between the silicon and silver paste produced a contact potential of about $0.15 \mathrm{~V} .^{32,33}$ There are two other reasons. First, the small potential change is caused by the $0.2 \mu \mathrm{A}$ leakage current in the system. Second, the contact potential is generated by the three-layer structure of the $\mathrm{Ag}$ electrode-silver paste-copper ${ }^{34}$ lead during the electrode welding (Fig. 3N). However, the potential generated with respect to the above-mentioned two reasons is very small. Because the internal $\mathrm{KCl}$ solution is in contact with the external solution through a nanochannel with a small diameter, the liquid junction potential is very small and can be excluded. This potential will be eliminated in further studies in the future. To show more details of the comparison between the micro RE potential and the glass tube RE potential, $185 \mathrm{mV}$ was added to the potential of the glass tube electrode when plotting the graph.

\subsection{Stability of miniaturized RE under different buffer concentrations}

To prove that the electrodes can be used in a complex field environment, a comparison test was carried out for the potential stability of the RE under different concentrations of electrolyte. The open-circuit voltage method was used to determine the change in the potential of the $\mathrm{RE}$ in different concentrations of a $\mathrm{Na}_{2} \mathrm{SO}_{4}$ solution at $\mathrm{pH}=7$. The concentration of the $\mathrm{Na}_{2} \mathrm{SO}_{4}$ solution ranged from $3 \mathrm{mM}$ to $30 \mathrm{mM}$, and the concentration gradient was $3 \mathrm{mM}$. Finally, the potential fluctuation of the miniaturized $\mathrm{RE}$ and glass tube $\mathrm{RE}$ at different electrolyte concentrations is shown in Fig. 6. Fortunately, Fig. 6 shows that the potential of the RE hardly drifts as the electrolyte concentration changes. Under 10 different concentrations of $\mathrm{Na}_{2} \mathrm{SO}_{4}$ solution, the reference potential of the miniaturized $\mathrm{RE}$ is $560.71 \mathrm{mV} \pm 1.314 \mathrm{mV}$. Under the same experimental conditions, the reference potential of the glass tube RE is $375.43 \mathrm{mV}$ $\pm 1.301 \mathrm{mV}$. According to the Nernst equation, ${ }^{27}$ the potential of the $\mathrm{RE}$ is affected by the temperature and chloride ion concentration. Obviously, the temperature and ion concentration are constant in this experiment. This potential fluctuation is probably caused by the liquid junction potential and systematic measurement error of $\mathrm{KCl}$ solution and $\mathrm{Na}_{2} \mathrm{SO}_{4}$ solution. This change in potential does not affect the results of the electrochemical experiments.

\subsection{Consistency of batch-microfabricated miniaturized RE}

In this study, micromachining technology was used to prepare the miniaturized RE. The ideal RE would have a smaller size, better stability, and consistency. The reference potentials of five different miniaturized REs manufactured in the same batch were measured using the open-circuit voltage method in a $3 \mathrm{mM}$ $\mathrm{Na}_{2} \mathrm{SO}_{4}$ solution. The results are shown in Table 1 . The stabilized potentials of miniaturized REs have good consistency: $0.534 \mathrm{~V}, 0.574 \mathrm{~V}, 0.559 \mathrm{~V}, 0.566 \mathrm{~V}$, and 0.586 V. These REs have the same $\mathrm{KCl}$ concentration $(3 \mathrm{M})$, temperature, and manufacturing process, but the leads are artificial and specific. Therefore, the difference in the reference potential can be attributed to the following: the lead contact uses silver paste as a conductive intermediate, and the degree of drying of the silver paste affects the magnitude of the contact potential. The impedance of the RE was used to approximately indicate the rate at which the internal chloride ions are exchanged with the external solution. The measured impedance of the miniaturized $\mathrm{RE}$ is $\mathbf{3 . 6}-\mathbf{1 7 . 4} \mathrm{k} \Omega$, which is higher than that of the commercial glass tube RE whose resistive impedance is $2.5 \mathrm{k} \Omega$, and also higher than that of a microporous polymer junction $\mathrm{RE}^{22}$ whose resistive impedance is $0.96 \mathrm{k} \Omega$.

\subsection{Application of RE in the determination of nutrients in water}

To verify the practicality of the novel silicon-base miniaturized $\mathrm{RE}$ in water, a non-integrated Au disk electrode was used as the WE, a non-integrated Pt electrode was used as the CE, and this miniaturized $\mathrm{Ag} / \mathrm{AgCl} \mathrm{RE}$ was used as the $\mathrm{RE}$ to construct a three-electrode system to detect the nitrite. In this experiment, 0.1 M PBS was selected as the buffer, and nitrite in water was detected by $\mathrm{CV}$. The concentration of nitrite was in the range from $800 \mu \mathrm{M}$ to $10 \mathrm{mM}$. The nitrite ions were oxidized to nitrate ions on the surface of the Au WE, which is accompanied by the movement of electrons. Therefore, the relationship between the current peak value on the CV curve and nitrite concentration is shown in Fig. 7A. Fig. 7A shows that the linearity between the peak current signal and nitrite concentration is good, and $R^{2}=$ 0.998. This indicates that the three-electrode system can accurately determine the nitrite content. This also shows that the new RE has good stability and accuracy for nitrite detection. To further verify the performance of the new RE, a glass tube RE, the above-mentioned $\mathrm{WE}$, and $\mathrm{CE}$ were used to form a threeelectrode system using the same method and experimental conditions, but the concentration range of nitrite was slightly changed to detect the nitrite content in water. Fig. 7B shows the linear relationship between the nitrite concentration and the peak of the CV curve in two cases, and their $R^{2}$ is 0.998 and 0.999. This indicates that the RE can be used for complex practical applications with better stability and durability, as shown in Section 3.2 . 
The stability and consistency of the batch-microfabricated miniaturized $\mathrm{Ag} / \mathrm{AgCl} \mathrm{RE}$ were compared with previously reported $\mathrm{Ag} / \mathrm{AgCl} \mathrm{REs}^{17,18,23,26}$ and were given in Table 2.

\section{Conclusions}

A novel batch-microfabricated silicon-base miniaturized $\mathrm{Ag}$ / AgCl (RE) was successfully developed. Tests show that the miniaturized RE has good stability, consistency, and is suitable for actual water pollution determination. This study provides a feasible technical solution for the design of electrochemical sensors for environmental water monitoring. We demonstrated that this miniaturized RE has the advantages of a small size, better reference potential stability, and performance consistency because of the batch fabrication technique. Moreover, because the fabrication of the RE is compatible with standard MEMS technology, it is expected that miniaturized multiparameter electrochemical sensors can be manufactured by integrating diverse WEs with this proposed RE. This novel strategy for the design and manufacturing of miniaturized REs has a bright future in the extensive use of electrochemical sensors for the online monitoring of water pollutants. However, two aspects should be improved: first, it should be ensured that the change in electrode potential with temperature and $\mathrm{KCl}$ solution concentration is in accordance with the Nernst equation. Second, the soldering of the sensor is still manual and specific, and the soldering method has a contact potential and affects the consistency of the electrode chip. These problems will be optimized in future studies.

\section{Conflicts of interest}

There are no conflicts to declare.

\section{Acknowledgements}

This work was financially supported by the grants received from the National Science Foundation of China (No. 61871243), Natural Science Foundation of Zhejiang Province (No. LGF18F010004 and No. LQ19F010004), and China postdoctoral Science Foundation (No. 2018M642384). This study was also sponsored by K. C. Wong Magna Fund in Ningbo University.

\section{Notes and references}

1 M. S. Han and D. H. Kim, Naked-eye detection of phosphate ions in water at physiological $\mathrm{pH}$ : a remarkably selective and easy-to-assemble colorimetric phosphate-sensing probe, Angew. Chem., 2002, 114(20), 3963-3965.

2 W. L. Daniel, M. S. Han, J. S. Lee, et al., Colorimetric nitrite and nitrate detection with gold nanoparticle probes and kinetic end points, J. Am. Chem. Soc., 2009, 131(18), 63626363.

3 Z. Zou, A. Jang, E. MacKnight, et al., Environmentally friendly disposable sensors with microfabricated on-chip planar bismuth electrode for in situ heavy metal ions measurement, Sens. Actuators, B, 2008, 134(1), 18-24.
4 G. Mills and G. Fones, A review of in situ methods and sensors for monitoring the marine environment, Sens. Rev., 2012, 32(1), 17-28.

5 C. D. M. Campos and J. A. F. da Silva, Applications of autonomous microfluidic systems in environmental monitoring, RSC Adv., 2013, 3(40), 18216-18227.

6 S. Kurbanoglu, M. A. Unal and S. A. Ozkan, Recent developments on electrochemical flow injection in pharmaceuticals and biologically important compounds, Electrochim. Acta, 2018, 287, 135-148.

7 K. M. Miranda, M. G. Espey and D. A. Wink, A rapid, simple spectrophotometric method for simultaneous detection of nitrate and nitrite, Nitric Oxide, 2001, 5(1), 62-71.

8 H. S. Lo, K. W. Lo, C. F. Yeung, et al., Rapid visual and spectrophotometric nitrite detection by cyclometalated ruthenium complex, Anal. Chim. Acta, 2017, 990, 135-140.

9 I. Vopálenská, L. Váchová and Z. Palková, New biosensor for detection of copper ions in water based on immobilized genetically modified yeast cells, Biosens. Bioelectron., 2015, 72, 160-167.

10 P. Biswas, A. K. Karn, P. Balasubramanian, et al., Biosensor for detection of dissolved chromium in potable water: a review, Biosens. Bioelectron., 2017, 94, 589-604.

11 M. Govindhan, B. R. Adhikari and A. Chen, Nanomaterialsbased electrochemical detection of chemical contaminants, RSC Adv., 2014, 4(109), 63741-63760.

12 D. Ning, H. Zhang and J. Zheng, Electrochemical sensor for sensitive determination of nitrite based on the PAMAM dendrimer-stabilized silver nanoparticles, J. Electroanal. Chem., 2014, 717, 29-33.

13 G. Amala and S. M. Gowtham, Recent advancements, key challenges and solutions in non-enzymatic electrochemical glucose sensors based on graphene platforms, RSC Adv., 2017, 7(59), 36949-36976.

14 A. K. Covington, J. V. Dobson and L. Wynne-Jones, The calomel electrode-I. Evaluation of the standard potential at $25^{\circ}$ and the activity coefficients of hydrochloric acid solutions, Electrochim. Acta, 1967, 12(5), 513-523.

15 I. Dendo, Precision silver/silver chloride electrodes// International Conference of the IEEE Engineering in Medicine \& Biology Society, IEEE, 1994.

16 M. J. De Giz, S. A. S. Machado, L. A. Avaca, et al., High area $\mathrm{Ni}-\mathrm{Zn}$ and Ni-Co-Zn codeposits as hydrogen electrodes in alkaline solutions, J. Appl. Electrochem., 1992, 22(10), 973977.

17 H. Suzuki, T. Hirakawa, S. Sasaki, et al., Micromachined liquid-junction $\mathrm{Ag} / \mathrm{AgCl}$ reference electrode, Sens. Actuators, B, 1998, 46(2), 146-154.

18 M. Ciobanu, J. P. Wilburn, N. I. Buss, et al., Miniaturized reference electrodes based on Ag/AgiX internal reference elements. I. Manufacturing and performance, Electroanalysis, 2002, 14(14), 989-997.

19 Z. Zhao, H. Tu, E. G. R. Kim, et al., A flexible Ag/AgCl micro reference electrode based on a parylene tube structure, Sens. Actuators, B, 2017, 247, 92-97.

20 I. Shitanda, M. Komoda, Y. Hoshi, et al., An instantly usable paper-based screen-printed solid-state $\mathrm{KCl} / \mathrm{Ag} / \mathrm{AgCl}$ 
reference electrode with long-term stability, Analyst, 2015, 140(19), 6481-6484.

21 A. Kisiel, H. Marcisz, A. Michalska, et al., All-solid-state reference electrodes based on conducting polymers, Analyst, 2005, 130(12), 1655-1662.

22 J. Pedrotti, L. Angnes and I. G. R. Gutz, Miniaturized reference electrodes with microporous polymer junctions, Electroanalysis, 1996, 8(7), 673-675.

23 M. A. G. Zevenbergen, G. Altena and V. A. T. Dam, et al., Solid state $\mathrm{pH}$ and chloride sensor with microfluidic reference electrode//Electron Devices Meeting (IEDM), 2016 IEEE International, IEEE, 2016, pp. 26.1.1-26.1.4.

24 R. Hayashi, K. Akio and Y. Hashizume, Reference electrode and a measuring apparatus using the same, US Pat. 5,037,527, 1991-8-6.

25 T. Rim, K. Kim, N. Hong, et al., Investigation of the electrical stability of Si-nanowire biologically sensitive field-effect transistors with embedded $\mathrm{Ag} / \mathrm{AgCl}$ pseudo reference electrode, RSC Adv., 2013, 3(21), 7963-7969.

26 I. Y. Huang, R. S. Huang and L. H. Lo, Improvement of integrated $\mathrm{Ag} / \mathrm{AgCl}$ thin-film electrodes by $\mathrm{KCl}$-gel coating for ISFET applications, Sens. Actuators, B, 2003, 94(1), 53-64.
27 D. J. G. Ives, G. J. Janz and C. V. King, Reference electrodes: theory and practice, J. Electrochem. Soc., 1961, 108(11), 246C$247 \mathrm{C}$.

28 D. Pletcher, A first course in electrode processes, Royal Society of Chemistry, 2009, pp. 8-13.

29 X. Han, X. Wang, X. Sun, et al., Nutrient distribution and its relationship with occurrence of red tide in coastal area of East China Sea, Yingyong Shengtai Xuebao, 2003, 14(7), 1097-1101.

30 J. Haneveld, H. Jansen, E. Berenschot, et al., Wet anisotropic etching for fluidic 1D nanochannels, J. Micromech. Microeng., 2003, 13(4), S62.

31 T. Rogers and J. Kowal, Selection of glass, anodic bonding conditions and material compatibility for silicon-glass capacitive sensors, Sens. Actuators, A, 1995, 46(1-3), 113-120.

32 E. H. Rhoderick, Metal-semiconductor contacts, IEE Proc., Part I: Solid-State Electron Devices, 1982, 129(1), 1.

33 W. Mönch, Metal-semiconductor contacts: electronic properties, Surf. Sci., 1994, 299, 928-944.

34 L. H. Fisher, Contact potentials between metals: history, concepts, and persistent misconceptions, Am. J. Phys., 1976, 44(5), 464. 